\title{
Characterization of ferromagnetic contacts to carbon nanotubes
}

\author{
D. Preusche, ${ }^{\text {a) }}$ S. Schmidmeier, E. Pallecchi, Ch. Dietrich, A. K. Hüttel, J. Zweck, and \\ Ch. Strunk \\ Institute for Experimental and Applied Physics, University of Regensburg, 93040 Regensburg, Germany
}

(Received 6 April 2009; accepted 14 August 2009; published online 23 October 2009)

\begin{abstract}
We present an investigation of different thin-film evaporated ferromagnetic materials for their suitability as electrodes in individual single-wall and multi-wall carbon nanotube-based spin devices. Various electrode shapes made from permalloy $\left(\mathrm{Ni}_{81} \mathrm{Fe}_{19}\right)$, the diluted ferromagnet PdFe, and $\mathrm{PdFe} / \mathrm{Fe}$ bilayers are studied for both their micromagnetic properties and their contact formation to carbon nanotubes. Suitable devices are tested in low-temperature electron transport measurements, displaying the typical tunneling magnetoresistance of carbon nanotube pseudo-spin valves. () 2009 American Institute of Physics. [doi:10.1063/1.3225571]
\end{abstract}

\section{INTRODUCTION}

Carbon nanotubes have been a widely investigated material for spintronics devices over the past years, which is above all due to their unique electrical properties, i.e., high Fermi velocity, quasi-one-dimensional ballistic transport, long spin lifetimes, and weak influence of nuclear spin. ${ }^{1,2}$ To obtain spin-dependent electron emission or absorption, a nanotube is typically contacted by ferromagnetic electrodes. Thin-film ferromagnets diluted with Pd are known to make a good electrical contact to individual carbon nanotubes, accessing the Kondo ${ }^{3,4}$ and Fabry-Perot regimes. ${ }^{5}$ Pd is used for its contact transparency to carbon nanotubes ${ }^{6}$ and its transgression into a ferromagnetic phase with even small admixture of a ferromagnet. ${ }^{7}$ For both multi-wall carbon nanotube (MWCNT) $)^{2,4,8,9}$ and single-wall carbon nanotube $(\mathrm{SWCNT})^{2,3,5,10}$ devices, the tunnel magnetoresistance $(\mathrm{TMR})^{11}$ has been observed. Any industrial application of the observed principles, however, will require a large degree of reproducibility and control, in particular, regarding the detailed micromagnetic structure of the nanotube contact electrodes.

This work aims at an improvement of the switching properties of the ferromagnetic electrodes, i.e., obtaining reliable reversal of the magnetization at reproducible coercive field values with a focus on PdFe alloys. Simultaneously, we monitor the electrical interface quality from ferromagnet to nanotube, including low-temperature magnetotransport measurements. Here, reliable control of magnetic switching is of particular importance as there are no direct means to check the magnetic domain structure once the sample is mounted in a cryostat for transport measurements.

We investigate a typical pseudo-spin valve geometry, ${ }^{5,8,9,12}$ as shown in Fig. 1(a) for a MWCNT and in Fig. 1(b) for a SWCNT. Two ferromagnetic electrodes F1 and $\mathrm{F} 2$ are designed such that they have a different coercive field. This is achieved here by making use of shape anisotropy, which can be tuned via the sample geometry. In magnetoresistance measurements, a sufficiently large external magnetic field is applied to saturate and align the magneti-

${ }^{a)}$ Electronic mail: dominik.preusche@ physik.uni-r.de. zation of both contacts parallel to it. A magnetic field sweep to opposite field direction will first switch the magnetization of the contact with smaller coercive field, thereby achieving antiparallel configuration, and then the magnetization of the second, resulting in a parallel configuration with polarity opposite to the initial one.

\section{LORENTZ MICROSCOPY IMAGING OF THE MAGNETIC SWITCHING OF FERROMAGNETIC ELECTRODES}

For carbon nanotube magnetotransport experiments, the ferromagnetic contact electrodes are required to have a difference in coercive field large enough to be resolvable in transport measurements, e.g., $10 \mathrm{mT}$ or more. A second requirement for reliable and reproducible magnetic switching is that the segment of the electrode contacting the carbon nanotube be in a well-defined single domain state to allow experimental control over injecting a spin-polarized current.

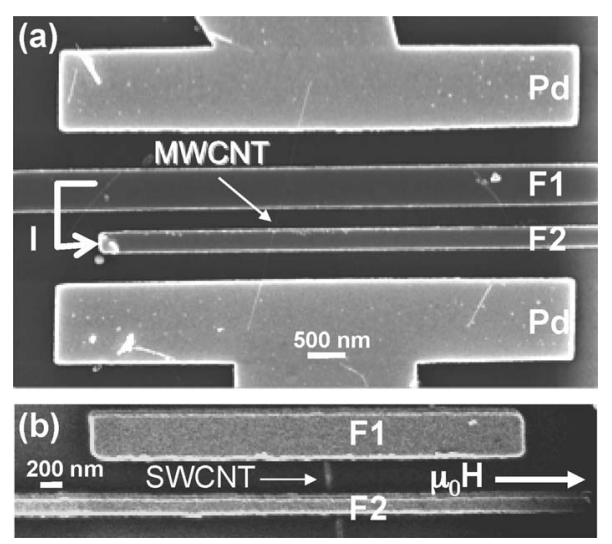

FIG. 1. (a) Scanning electron microscope (SEM) image of a MWCNT contacted by two ferromagnetic electrodes, forming a pseudo-spin valve device. The electrodes $\mathrm{F} 1$ and $\mathrm{F} 2$ are fabricated from permalloy $\left(\mathrm{Py}\right.$ or $\left.\mathrm{Ni}_{81} \mathrm{Fe}_{19}\right)$; in addition two nonferromagnetic Pd electrodes to allow four-terminal measurements are shown. (b) SEM image of a SWCNT contacted by two ferromagnetic electrodes $\mathrm{F} 1$ and $\mathrm{F} 2$ made from the dilute ferromagnet $\mathrm{Pd}_{60} \mathrm{Fe}_{40}$. An arrow indicates the direction of an externally applied magnetic field. Because of shape anitrosopy, the different electrode aspect ratios result in a differing coercive field. 


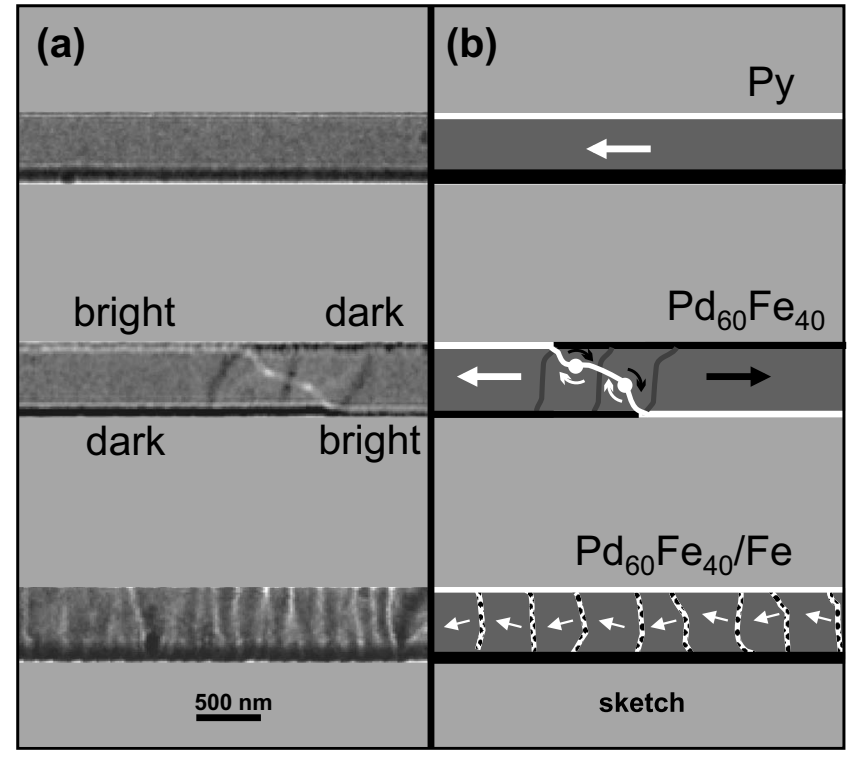

FIG. 2. (a) Comparison of Lorentz microscopy images of rectangular electrodes made from different ferromagnetic materials. An external magnetic field is tuned close to the coercive field. All strips have lateral dimensions of $500 \mathrm{~nm} \times 16 \mu \mathrm{m}$; the film thickness is $45 \mathrm{~nm}$. (b) Sketch pointing out the observed micromagnetic features. The magnetization direction (big black and white arrows) can be determined by the position of the dark and bright contrast. Solid black and white lines, as seen in the $\mathrm{Pd}_{60} \mathrm{Fe}_{40}$ strip, are caused by magnetic domain walls with full magnetization reversal. Cross-tie domain walls (gray lines) enclose a magnetic vortex (white dot with curved white and black arrows). Weak contrast lines, drawn dotted in the sketch, correspond to ripple domain walls with only small changes in magnetization direction (small white arrows).

Transmission electron microscopy (TEM) in Lorentz microscopy mode provides a powerful tool to investigate these properties of thin ferromagnetic films.

\section{A. Methods}

Lorentz microscopy ${ }^{13-15}$ allows direct observation of the magnetic domain structure of a ferromagnetic specimen and its evolution in an external magnetic field by sending a parallel (out of focus) electron beam through a magnetic specimen. The deflection of the beam due to the Lorentz force can be visualized by defocusing the objective lens. At the walls enclosing a magnetic domain, the electrons are deflected as they transverse the ferromagnetic film and form either a convergent or a divergent set of partial electron waves. Correspondingly, an increase or a decrease in the electron beam intensity is detected at the location of domain walls. It should be noted that not only domain walls but also other variations in the magnetic induction within the specimen can be visualized by this technique.

If the specimen is magnetized homogeneously, every transmitted electron will be deflected to the same side. In this case, a brightly contrasted feature forms on the edge of the specimen where the deflected beam through it overlaps with the undeflected beam passing next to it. On the opposite side, the deflection of the electrons will partially deplete the edge region which manifests in a dark contrast line on the detector. These dark and bright contrast features can, for instance, be seen within the permalloy strip shown in the topmost panel of Fig. 2. Magnetic domain walls, where spins oppose each other in frustration, appear as dark or bright features within the strip area (see middle panel of Fig. 2). A detailed discussion of Fig. 2 will be given below.

Driving the electron beam focus from above to below the sample plane reverses the Lorentz-force induced contrast, providing a consistency test as to whether observed features are indeed of magnetic origin. A magnetization reversal due to an external magnetic field can be detected by observing subsequent images during a field sweep when a structure's edge contrast is inverted. Note that on the borders of the observed ferromagnetic structures, the dark Lorentz contrast appears more pronounced than the bright one. This is partly owed to a superposition with a bright contrast all around the observed structures stemming from Fresnel edge diffraction. The magnetization structure can therefore be read off most clearly by tracing structures of dark edge contrast.

In the TEM used for the work at hand, an external magnetic field $\mu_{0} H$ can be applied only parallel to the electron beam, i.e., perpendicular to the sample plane. An in-plane field component $\mu_{0} H_{\|}=\mu_{0} H \sin \alpha$ with respect to the sample plane can be tuned by maintaining this field at constant magnitude and tilting the sample by an angle $\alpha$. At a maximum tilt angle of $\pm 25^{\circ}$, the in-plane component reaches about half the value of the out-of-plane component. Rotating the sample and thereby sweeping the in-plane field from saturation through zero field to saturation in opposite direction allows to monitor the magnetization reversal process of the ferromagnetic specimen.

Electron transmission microscopy requires samples to be prepared on a grid or thin film membrane with low electron beam contrast. The metal test structures were patterned by electron beam lithography (EBL) and thermally evaporated in vacuum onto $50 \mathrm{~nm}$ thin low-stress plasma-enhanced chemical vapor deposition silicon nitride membranes. Owing to shape anisotropy, Néel walls, i.e., with the frustrated spins being confined to the film plane, occur in sufficiently thin ferromagnetic films. Above a material-specific thickness, which is smaller for weaker magnetization, Bloch walls dominate. Here, the spins form domain walls by gradually turning out of the film plane. According to our experience, the threshold thickness of permalloy $\left(\mathrm{Py}, \mathrm{Ni}_{81} \mathrm{Fe}_{19}\right)$ films is about $50 \mathrm{~nm}$. We therefore keep the film thicknesses below that value. Also the large aspect ratio of film thickness compared to the lateral electrode dimensions is expected to favor an in-plane orientation of the magnetization. ${ }^{16}$ We hence start our discussion with the effect of this in-plane magnetic field component. In any case, effects of magnetization and magnetic field components parallel to the electron beam are not imaged in Lorentz microscopy due to the cross product in the Lorentz force.

\section{B. Material dependence of the domain structure and magnetization reversal properties}

The investigated materials are chosen for their magnetic properties or expected contact transparency to carbon nanotubes. We consider permalloy $\left(\mathrm{Py}, \mathrm{Ni}_{81} \mathrm{Fe}_{19}\right)$ and the giant paramagnet Pd diluted with $\mathrm{Fe}$, as we expect diluted ferromagnetic PdFe alloys to combine the benefits of the strong 
ferromagnetism of iron and the transparent contacts of palladium-carbon nanotube interfaces. ${ }^{2,6}$ The composition of the PdFe alloy is adjusted by setting the evaporation rates from two confocal thermal evaporation sources appropriately. In addition, magnetic bilayer structures with $10 \mathrm{~nm}$ of $\mathrm{Pd}_{60} \mathrm{Fe}_{40}$ and a 35-nm-thick Fe layer-to stabilize the magnetization of the magnetically soft PdFe by the magnetically hard $\mathrm{Fe}$ - are discussed. To prevent the iron from oxidation and for improved interface resistance with the Pd leads, these bilayers were additionally capped with $5 \mathrm{~nm}$ of $\mathrm{Pd}$.

In Fig. 2(a), rectangular strips of equal dimensions made from these three polycrystalline materials are compared. To allow comparison of the domain structure in different ferromagnets, the Lorentz microscopy images are taken at the field values close to magnetization reversal in the specific material. We concentrate initially on the central segment of the strip, where the contacted carbon nanotube would be placed in transport measurements. The majority spin orientation and magnetic domain structure of this portion determine spin orientation and polarization of an injected current. The sketches of Fig. 2(b) point out the relevant magnetic features of the Lorentz images.

\section{Permalloy}

Permalloy (Py) was chosen as a ferromagnetic material for its high magnetic permeability, low coercive field, and large magnetic anisotropy. ${ }^{16-18}$ From the homogeneity of the entire Py strip in Fig. 2(a) it can be concluded that the observed segment is in a single domain state. In the Lorentz image the magnetic domain appears bordered by a continuous bright contrast line on the top side and a dark one on the bottom side. The corresponding arrow in Fig. 2(b) symbolizes this uniform magnetization orientation of the single domain. Note that the absolute direction of the magnetization can only be determined from the dynamics during a full magnetic sweep by comparing subsequent Lorentz images.

\section{PdFe alloy}

In contrast to $\mathrm{Py}$, the $\mathrm{Pd}_{60} \mathrm{Fe}_{40}$ strip segment in Fig. 2(a) is in a two-domain state at a small field increase beyond magnetization reversal but not yet saturated. The black and white arrows in the sketch of Fig. 2(b) highlight the opposite magnetization orientation of the domains. The border contrast changes from bright to dark where the sharp white line connects to the strip boundary. In addition, three darkcontrasted, roughly parallel lines can be made out crossing the bright line. Such so-called cross-tie wall structures are typical for samples between Néel and Bloch phase ${ }^{16}$ where a successive row of in-plane (Néel) and out-of-plane (Bloch) type walls occurs. For our polycrystalline specimen, we expect a vanishing crystalline anisotropy, so the magnetic dipolar (or shape) anisotropy is dominant. Due to the small magnetization of $\mathrm{Pd}_{60} \mathrm{Fe}_{40}$ compared to stronger ferromagnetic materials, also the shape anisotropy is small. This means that even low magnetic fields perpendicular to the film can lead to relatively large normal components $M_{z}$ of magnetization. In any case, magnetic multidomain and crosstie wall configurations of the $\mathrm{Pd}_{60} \mathrm{Fe}_{40}$ strip render its mag-

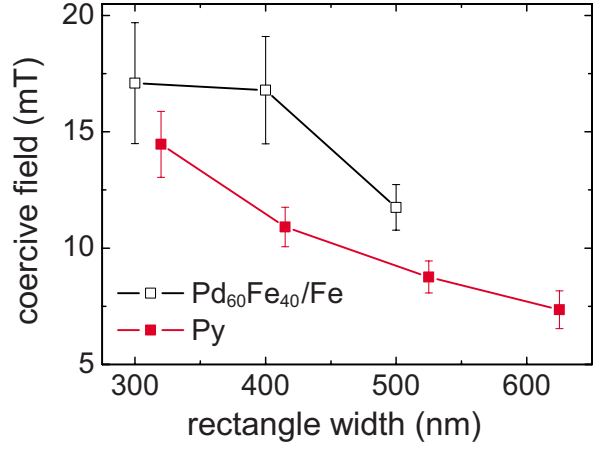

FIG. 3. (Color online) Comparison of the coercive field of Py and $\mathrm{Pd}_{60} \mathrm{Fe}_{40} / \mathrm{Fe}$ strips. Data points are retrieved from room temperature Lorentz microscopy measurements and averaged over three strips of same dimensions fabricated in the same EBL and metallization process. While the absolute switching field is higher for strips of $\mathrm{Pd}_{60} \mathrm{Fe}_{40} / \mathrm{Fe}$ than of $\mathrm{Py}$, the deviation from strip to strip is much larger. Py displays a more reliable switching (smaller error bars).

netization configuration ill-defined for the operation of a CNT pseudo-spin valve. Furthermore, the specific domain wall pattern is often different for any two magnetic sweeps. Occurrence of magnetoresistance in a pseudo-spin valve fabricated from this material is therefore prone to a random domain configuration and the position of magnetic pinning centers.

\section{PdFe alloy with an Fe magnetization stabilization layer}

The $\mathrm{Pd}_{60} \mathrm{Fe}_{40} / \mathrm{Fe}$ bilayer strips display a more uniform overall magnetization, as indicated by a continuous dark, respectively bright, contrast along the entire borderlines of the strip. In addition, a fine structure appears in Fig. 2(a), which signals the presence of ripple domains, i.e., areas in which the spins deviate from the overall magnetization direction by a small angle as sketched in the bottom structure of Fig. 2(b). The formation of ripple domains is a well-known phenomenon for thin film materials with a high saturation magnetization like $\mathrm{Fe},{ }^{16}$ suggesting that the Fe layer dominates the magnetic properties of the strip.

Figure 3 compares the switching behavior of $\mathrm{Pd}_{60} \mathrm{Fe}_{40} / \mathrm{Fe}$ and Py strips of equal lateral dimension and total thickness of $45 \mathrm{~nm}$. While the $\mathrm{Pd}_{60} \mathrm{Fe}_{40} / \mathrm{Fe}$ magnetic bilayer switches at higher fields than permalloy, it does so with a much higher margin of error.

\section{Shape dependence of the coercive field}

Next, we compare the magnetic switching behavior of rectangular, needle-shaped, ${ }^{19,20}$ and spoon-shaped ${ }^{21}$ electrode structures in order to identify the optimal geometry parameters for magnetic switching reproducibility and controllability. It is well known that by choice of very high aspect ratio of length to width, the smaller of the two parameters dominates the switching behavior via shape anisotropy. By setting the length to $16 \mu \mathrm{m}$ for all structures, investigations can be reduced to varying the electrode widths in the range from 200 up to $\sim 1 \mu \mathrm{m}$. Lorentz microscopy allows to determine whether a structure is of suitable dimensions to be in a single domain configuration. 


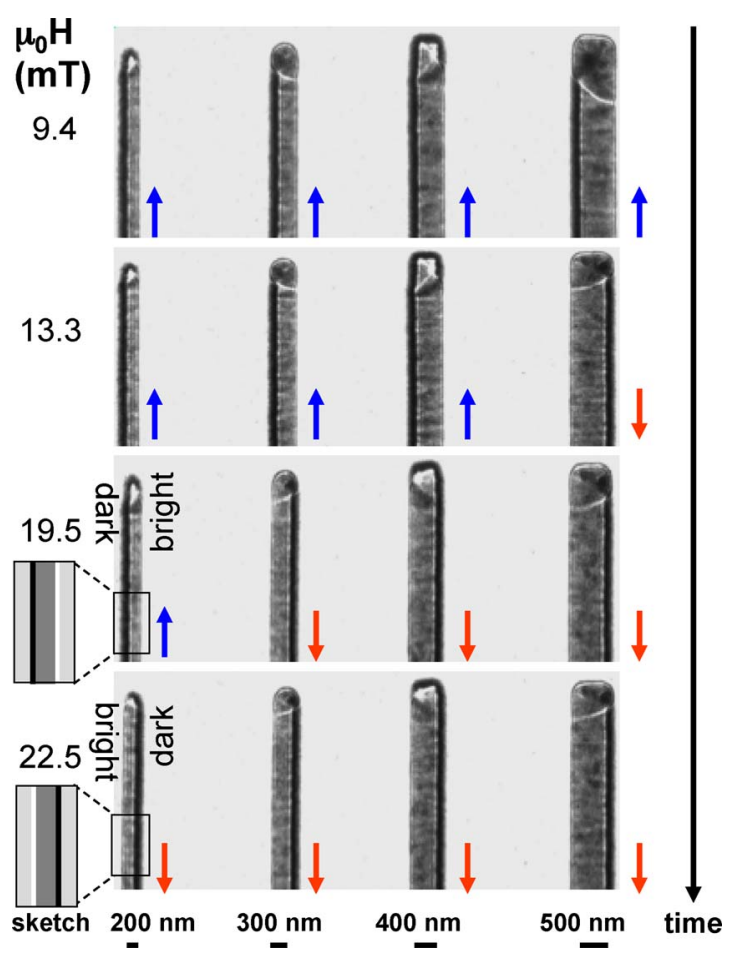

FIG. 4. (Color online) Lorentz microscopy images of four $\mathrm{Pd}_{60} \mathrm{Fe}_{40} / \mathrm{Fe}(10$ $\mathrm{nm} / 35 \mathrm{~nm}$ thick) strips of different widths $(200,300,400$, and $500 \mathrm{~nm})$. The length $(16 \mu \mathrm{m})$ is chosen large enough for the width only to govern the shape anisotropy. The strips have been fully saturated in a magnetic field parallel to the long strip axis (blue arrows, top row). When applying an increasing external field in opposite direction (subsequent lower image rows), magnetic switching takes place, see the red and blue upward arrows next to the strips. The required coercive field clearly decreases with increasing strip width. The black arrow indicates how images were taken subsequently in time during a single magnetic field sweep. Insets: sketches of the observed edge features, cf. Fig. 2.

\section{Rectangles}

Figure 4 shows four $\mathrm{Pd}_{60} \mathrm{Fe}_{40} / \mathrm{Fe}$ contact strips of different widths at different values of the external magnetic field $\mu_{0} H_{\|}$. Coming from saturation, where all strip magnetizations are aligned along $-\mu_{0} H_{\|}$(blue upward arrows, top image row), the external field is swept to opposite field direction $\mu_{0} H_{\|}$. As indicated by the arrow on the edge, subsequent image rows are recorded at increasing field values during a magnetic field sweep. The images show that the rectangular strips flip their magnetization consecutively at coercive fields increasing in order of decreasing strip width.

An interesting feature visible in Fig. 4 is the formation of a magnetic end domain at the upper end of the electrode. Its evolution can be monitored by following the magnetic features at increasing field values. It indicates that the magnetic end domains nucleate the magnetic switching of the whole strip. Otherwise, the strips all show a clear single magnetization direction as desired for a pseudo-spin valve contact electrode.

\section{Needle-shaped structures}

In Fig. 5, three $\mathrm{Pd}_{60} \mathrm{Fe}_{40} / \mathrm{Fe}$ needle-shaped structures of different widths are Lorentz imaged. A magnetization reversal analogous to Fig. 4 is found. Compared to rectangles, switching in needles is also governed by the structure width

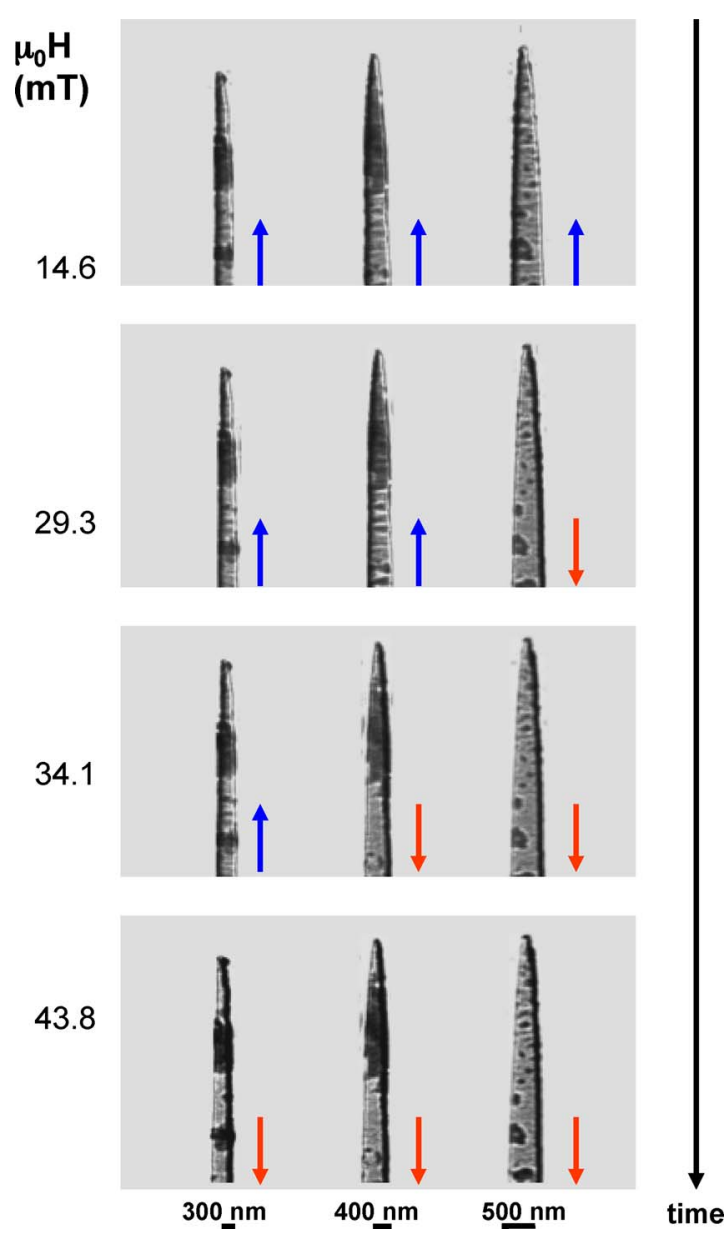

FIG. 5. (Color online) Lorentz microscopy images detailing the magnetization reversal of needle-shaped $\mathrm{Pd}_{60} \mathrm{Fe}_{40} / \mathrm{Fe}(10 \mathrm{~nm} / 35 \mathrm{~nm})$ structures differing in width $(300,400$, and $500 \mathrm{~nm})$ analogous to the data of Fig. 4. From upper to lower image row, an increasing magnetic field directed opposite to the original saturation magnetization is applied. The irregular spots on the image stem from nonmagnetic process contamination.

but occurs at higher coercive field. This may be explained by fewer magnetically frustrated spins within the pointed structure tips, i.e., fewer and smaller end domains, see Fig. 5 compared to the rectangles of Fig. 4. As the magnetization reversal is initiated by these end domains, their suppression also should translate into higher magnetization stability.

\section{Spoon-shaped structures}

In Fig. 6, TEM Lorentz microscopy images of spoonshaped structures, i.e., rectangular strips with attached disk, are presented. The disk diameter dependence of the magnetization reversal of attached rectangular strips is investigated. All strips have equal dimension, only the disk diameters are varied.

In a disk made from a ferromagnetic material, the spins can align in a vortex configuration. ${ }^{16}$ Depending on whether the vortex chirality is clockwise or counterclockwise, the vortex core appears in the Lorentz image as either a dark spot (structure in the first column of Fig. 6) or as a bright spot (second column). At zero external magnetic field, the vortex core is near the center of the disk. An external magnetic field along the strip "shoves" the bright (dark) vortex core to the right-hand side (left-hand side). 


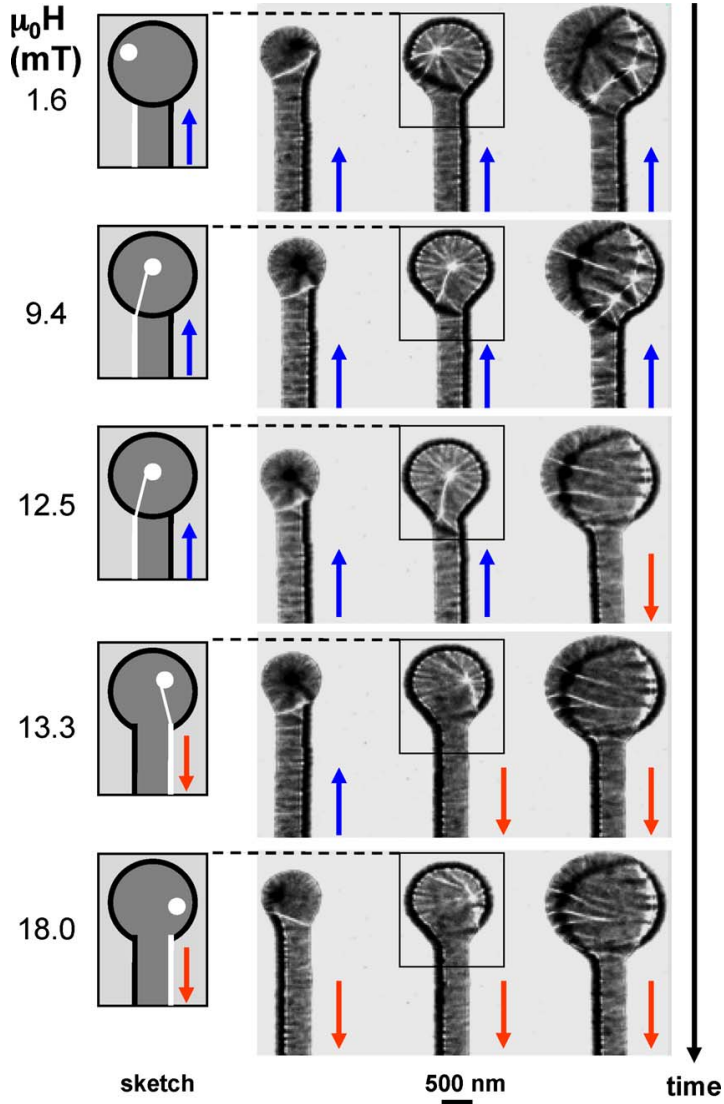

FIG. 6. (Color online) Lorentz microscopy images showing the magnetization reversal of three spoon-shaped structures. Each of the identical $16 \mu \mathrm{m} \times 500 \mathrm{~nm}, \mathrm{Pd}_{60} \mathrm{Fe}_{40} / \mathrm{Fe}$ rectangular strips has a disk-shaped structure of different diameters $(1.0,1.5$, and $2.0 \mu \mathrm{m})$ attached to its upper end. The lower part of the strip (not shown, at large distance) has a rectangular ending. Inside the disk, the contrast structure indicates the formation of a magnetic vortex. The magnetic switching of the strip with increasing external field (descending in image rows) is triggered when a domain wall originating from this vortex is driven into the rectangle.

This behavior can be observed most clearly in the example of the second column of Fig. 6, where the magnetization reversal of a "spoon" with a $1.5 \mu \mathrm{m}$ diameter disk is shown. A domain wall, seen as a bright line in the images, originates from the vortex core for all magnetic field values. Sweeping up from negative values of $\mu_{0} H$, the vortex core is still shifted at $+1.6 \mathrm{mT}$ to the left-hand side, owing to the magnetic remanence of the structure. The domain wall ends at the left-hand side of the disk. A field of $9.4 \mathrm{mT}$ is required to counter the magnetic remanence so the vortex is moved to the disk center. The white domain wall ends now near the joining of disk and strip as the domain to its left is expanding under the effect of the increasing magnetic field. At $12.5 \mathrm{mT}$ the vortex core is shifted to the right-hand side and the domain left to the said white domain wall is on the brink of expanding into the rectangular strip beneath; a small increase to $13.3 \mathrm{mT}$ is sufficient to cause the domain wall to pass through the rectangular strip, thereby initiating a magnetization reversal. At $18.0 \mathrm{mT}$, the vortex core is almost "pushed" out of the disk and the entire spoon-shaped structure is saturated. The switching field of the total spoon structure is lowered when the disk diameter is increased. We conclude that the disk can thus trigger the magnetization switching of the

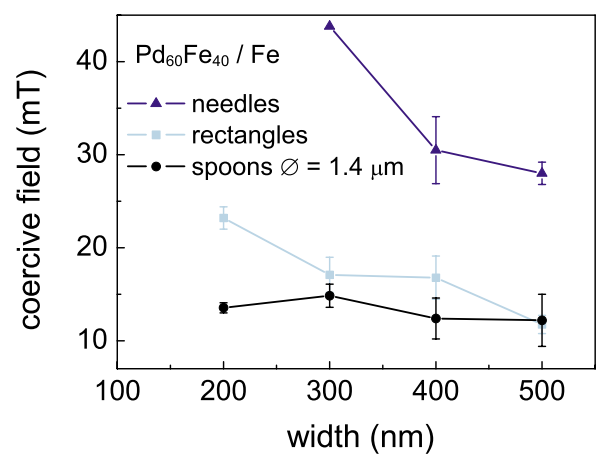

FIG. 7. (Color online) Shape influence on coercive field. The coercive field obtained from Lorentz microscopy images is plotted for spoon-shaped, rectangular, and needle-shaped structures made from $\mathrm{Pd}_{60} \mathrm{Fe}_{40} / \mathrm{Fe}$. In the case of the spoon shapes, the $16 \mu \mathrm{m}$ long rectangular "handles" are topped with identical disks of diameter $1.4 \mu \mathrm{m}$. For all structures, the (handle) strip width is varied ( $x$-axis in the graph). Coercive field values are averaged over four magnetic field sweeps of the same structure and shown with respective error bars. The coercive field of the needles shows the strongest increase with decreasing width. Whereas the rectangles still display a sizeable strip width dependence of the coercive field, the disk dominates the magnetic switching of the spoon structures, nearly equalizing the coercive field for all strip widths.

strip structure in a controlled way, independent of random end domains. However, as can be seen in the third column of Fig. 6, at large disk diameters no clear vortex forms but strong ripple domain structures typical for Fe thin films appear, ${ }^{22}$ counteracting the expected stabilizing effect on magnetic switching.

Both effects are illustrated by the data in Fig. 7, where we plot the coercive fields of strips ranging from 200 to 500 $\mathrm{nm}$ with and without an attached disk of diameter of $1.4 \mu \mathrm{m}$ or a needle tip. The disk clearly dominates the switching of the spoon-shaped structures and equals the coercive field of all strips around $12.5 \mathrm{mT}$. However, for larger structures a stronger scatter in measured values can be observed. The needles show the strongest width dependence on the coercive field.

\section{Effect of ferromagnetic supply lines}

Fabricating contact electrodes together with supply lines and bond pads in a single metallization process would simplify chip processing significantly. To investigate the effect of attaching large ferromagnetic supply lines on the magnetization reversal of the contact electrodes, two identical $\mathrm{Pd}_{60} \mathrm{Fe}_{40} / \mathrm{Fe}$ strips with and without attached typical supply lines and bond pad (not shown) geometry attached are compared in Fig. 8. To reduce the magnetic coupling between electrode and supply lines a disk is placed at their junction. This disk typically forms a strongly diameter-dependent vortex, as discussed above.

Coming from saturation at high negative field, at $0 \mathrm{mT}$ (left panel in Fig. 8) both contacts have their magnetization aligned downward (red arrows). At $7.1 \mathrm{mT}$, initiated by edge domains, the strip without attachment aligns along the external field (blue arrow). The strip with attachment follows at a coercive field of $10.2 \mathrm{mT}$ - in this particular case at a higher rather than at lower field value, as also observed with an attached disk structure. Still, there is a difference in coercive 


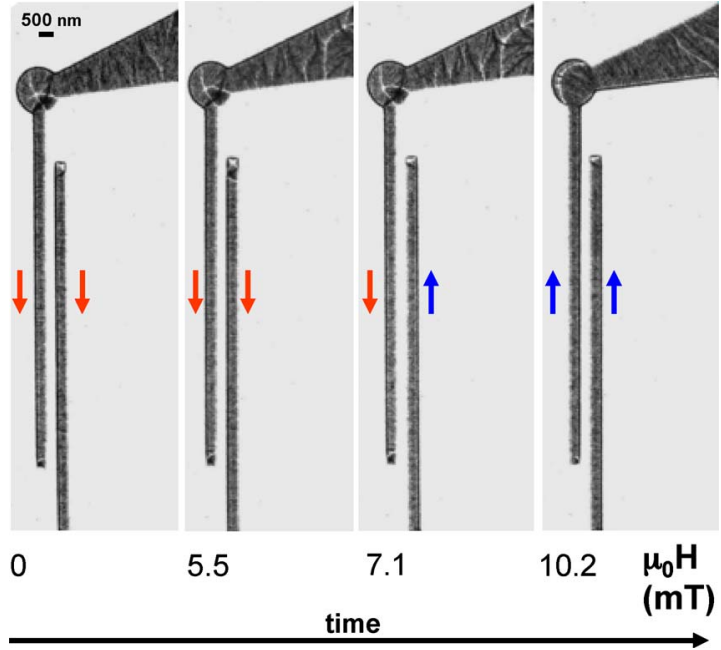

FIG. 8. (Color online) Lorentz microscopy images of a detached rectangular electrode compared to an electrode with attached large-area ferromagnetic leads. The electrode width is in both cases $500 \mathrm{~nm}$. Following the images from left to right, it can be observed how an external magnetic field initiates a magnetization reversal. The ferromagnetic leads change the switching field since the magnetization reversal can be initiated by the increasing external field driving a domain wall from the lead into the rectangular electrode.

field of $3.1 \mathrm{mT}$ indicating that the magnetization reversal is triggered by the multidomain configuration in the supply lines. Switching is rendered irreproducible due to the random domain structure in the bulky supply line.

\section{Temperature dependence}

Low temperatures are still an important prerequisite to many fundamental studies of spintronics devices. This raises the question whether the results obtained by room temperature Lorentz microscopy remain valid in the low-temperature regime. For this purpose, Fig. 9 compares the coercive fields of rectangular Py strips obtained at room temperature with those at $100 \mathrm{~K}$, the lowest accessible temperature in our TEM sample holder. Coercive field values agree, within the margin of error, for both temperatures, justifying an extrapolation of our findings to the low-temperature limit.

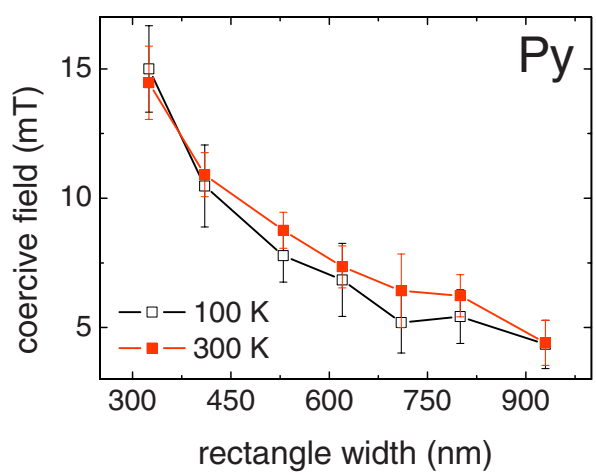

FIG. 9. (Color online) Comparison of the coercive field of rectangular Py strips of different widths and with a length of $16 \mu \mathrm{m}$ at room temperature $(300 \mathrm{~K}$, red) and close to liquid nitrogen temperature (100 K, white). Data points are retrieved from Lorentz microscopy measurements, averaging over three rectangular Py strips of same dimension fabricated in the same EBL and metallization process.

\section{APPLICATION IN MAGNETOTRANSPORT MEASUREMENTS}

For electronic transport experiments, we have fabricated SWCNT samples with rectangular electrodes made from $\mathrm{Pd}_{60} \mathrm{Fe}_{40}$ as well as MWCNT samples with electrodes made from $\mathrm{Pd}_{80} \mathrm{Fe}_{20}$, Py, and a $\mathrm{Pd}_{60} \mathrm{Fe}_{40} / \mathrm{Fe}$ magnetic bilayer. The magnetoresistance was measured both in an in-plane magnetic field along the electrodes and also in a perpendicular, out-of-plane field.

\section{A. Fabrication of nanotube devices}

SWCNTs have been grown by chemical vapor deposition (CVD) on highly $p$-doped Si wafers with $300 \mathrm{~nm}$ thermal oxide. The substrate can serve as backgate electrode. As feed gas we use a mixture of methane and hydrogen at $900{ }^{\circ} \mathrm{C}$ with $\mathrm{Fe}\left(\mathrm{NO}_{3}\right)_{3} \cdot 9 \mathrm{H}_{2} \mathrm{O}, \mathrm{Al}_{2} \mathrm{O}_{3}$, and $\mathrm{MoO}_{2}(\text { acac })_{2}$ as catalyst. ${ }^{23}$ Best results were obtained with a $\mathrm{CH}_{4}$ flux of 1.5 sccm (sccm denotes cubic centimeter per minute at STP) and a $\mathrm{H}_{2}$ flux of $0.23 \mathrm{sccm}$ although these parameters seem to be specific for the individual setup.

For the purposes of fabricating an individual SWCNT device, defect-free, clean nanotubes were located with respect to prepatterned alignment markers using either scanning electron or atomic force microscopy. In addition, high purity arc-discharge grown MWCNTs with typical diameters around $10 \mathrm{~nm}$ were dispersed on wafers from solution and localized similarly. The fabrication of contact electrodes was adapted to the nanotube location by EBL and thin film deposition.

\section{B. Room-temperature interface resistance of different ferromagnetic electrode materials to carbon nanotubes}

While the magnetic domain structure of the contact electrodes is one defining factor for the performance of a CNT pseudo-spin valve device, the nanotube contact resistance is another important material selection criterion.

A severe disadvantage of Py electrodes was their lack in interface quality to carbon nanotubes. In most devices, contact resistances of several megaohms were observed. Annealing for about $60 \mathrm{~s}$ at $500{ }^{\circ} \mathrm{C}$ and $10^{-4}$ mbar improved the room temperature resistance of MWCNT samples temporarily; applying, to the same end, a large bias voltage of 2-10 $\mathrm{V}$ led to a significant drop of the resistance of the tube-metal interface. However, the interface deteriorated again on a scale of several days. Even while temporarily decreased the sample resistance was marked by strong fluctuations and abrupt jumps.

Palladium is known to form high-transparency contacts to carbon nanotubes. ${ }^{6}$ Correspondingly it has also been shown that palladium-diluted ferromagnetic materials inherit this property. ${ }^{3,5,10,12}$ Yet we found that even four electrodes patterned on the same tube in the same lithography and $\mathrm{Pd}_{60} \mathrm{Fe}_{40}$ evaporation process can have highly different room temperature contact resistances, ranging from $20 \mathrm{k} \Omega$ to the megaohm range. While some CNT devices with PdFe contacts yielded transparent contacts to carbon nanotubes, e.g., 


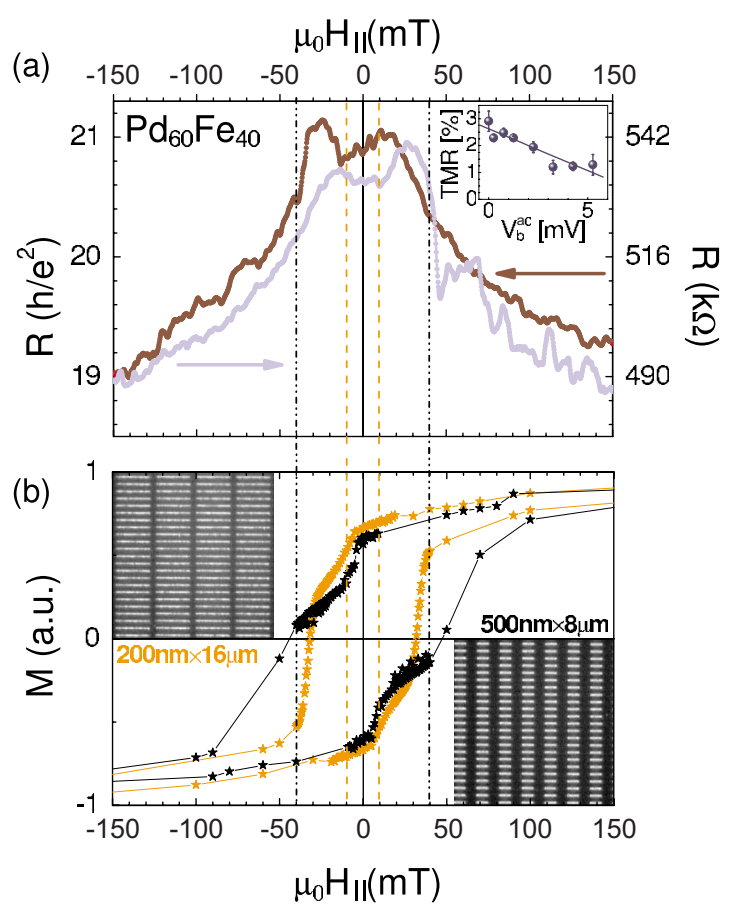

FIG. 10. (Color online) (a) Differential two-terminal resistance $R=d V / d I$ of a SWCNT pseudo-spin valve device measured with lock-in techniques at 4.2 $\mathrm{K}$ as a function of an in-plane magnetic field $\mu_{0} H_{\|}$along the electrode long axis. The ac bias was $3 \mathrm{mV}$. A TMR feature of $1.8 \%$ appears in upsweep (light blue curve) and downsweep (dark red curve) in the intervals of $\pm[10$ $\mathrm{mT}, 40 \mathrm{mT}]$. The wide $(500 \mathrm{~nm} \times 8 \mu \mathrm{m}$, marked in dark green) strip switches at smaller field than the narrow $(200 \mathrm{~nm} \times 16 \mu \mathrm{m}$, marked in bright orange) $\mathrm{Pd}_{60} \mathrm{Fe}_{40}$ strip. Dashed bright orange and dark green lines mark the magnetization switching of the narrow and wide electrodes. Inset: TMR as function of applied ac bias. (b) Magnetization hysteresis $M\left(\mu_{0} H_{\|}\right)$ of large arrays of $\mathrm{Pd}_{60} \mathrm{Fe}_{40}$ strips with identical dimensions as the contacts in (a), detected with a SQUID at $T=2 \mathrm{~K}$. The saturation magnetization is normalized to 1 and corresponds to $1.5 \times 10^{-4} \mathrm{emu}$ for the narrow strip array (bright orange) and $0.75 \times 10^{-4} \mathrm{emu}$ for the wide strip array (dark green). Insets: SEM images of the samples.

accessing the Kondo regime, the controllability of the magnetic domain configuration in this material remains insufficient.

In order to preserve the benefit of the low-resistive PdFe-CNT interface and to simultaneously obtain strong inplane magnetization, the aforementioned magnetic bilayer films consisting of $10 \mathrm{~nm} \mathrm{Pd}_{60} \mathrm{Fe}_{40}$ and a 35-nm-thick $\mathrm{Fe}$ layer were used. The contacting of carbon nanotubes with $\mathrm{Pd}_{60} \mathrm{Fe}_{40} / \mathrm{Fe}$ electrodes turned out to be less reliable than with $\mathrm{Pd}_{60} \mathrm{Fe}_{40}$ only. Resistances were typically in the megaohm range. With an annealing step, the resistance of some samples could be reduced to room temperature values of down to $120 \mathrm{k} \Omega$. The noise level was high compared to the other two investigated contact materials. Characteristic for these samples was the occurrence of random telegraph noiselike resistance jumps, exceeding any magnetoresistance features by a factor of typically $2-3$.

\section{Low-temperature magnetoresistance measurements on SWCNTs}

\section{TMR in a parallel magnetic field}

Figure 10(a) shows the two-terminal resistance of a SWCNT pseudo-spin valve with $\mathrm{Pd}_{60} \mathrm{Fe}_{40}$ rectangular elec-

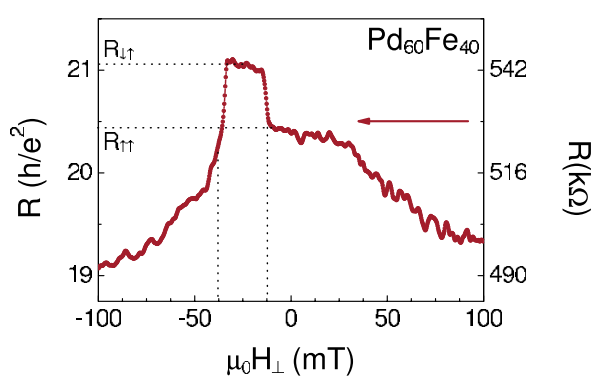

FIG. 11. (Color online) Differential two-terminal resistance $R=d V / d I$ of the same SWCNT device as in Fig. 10 at $4.2 \mathrm{~K}$, this time applying an out-ofplane magnetic field $B_{\perp}$ and an ac bias of $3 \mathrm{mV}$. TMR features of $3 \%$ appear in downsweep of the magnetic field within the interval of [10 $\mathrm{mT}, 40 \mathrm{mT}]$.

trodes. It is plotted as a function of the external magnetic field during a field upsweep (light blue curve) and downsweep (dark red curve) from saturation of the electrodes to saturation in opposite field direction. The external magnetic field was aligned along the length of the electrode strips. On top of background features, a positive TMR signal emerges as a steplike increase in resistance within an interval of $\pm[10$ $\mathrm{mT}, 40 \mathrm{mT}]$. It arises from the relative magnetization orientation of narrow and wide electrodes.

The amplitude of the tunneling magnetoresistance $\mathrm{TMR}=\left(R_{\uparrow \downarrow}-R_{\uparrow \uparrow}\right) / R_{\uparrow \uparrow}$ was inferred from measurements of the two-terminal resistances $R_{\uparrow \downarrow}$ and $R_{\uparrow \uparrow}$ in parallel and antiparallel magnetization orientations of the two ferromagnetic electrodes. We obtain a TMR effect of up to $3 \%$ on top of a larger background magnetoresistance that is probably due to electron-magnon scattering. In some cases we observed a less significant maximum before reaching zero field, see, e.g., the downsweep in Fig. 10(a). Its origin remains unclear at present. However, it seems to be absent when applying an out-of-plane field, compare Fig. 11.

The inset of Fig. 10(a) shows that the TMR drops with increasing the applied dc bias. This is to be expected because in addition to the increased current, the differential conductance $d I / d V \equiv I_{\text {ac }} / V_{\text {ac }}$ is effectively averaged over a larger source-drain voltage $V_{\text {sd }}$ window. ${ }^{5}$

\section{Magnetization reversal from SQUID measurements of ferromagnetic strips}

To confirm that the observed magnetoresistance features indeed originate from magnetic switching of the electrodes, we determined the coercive fields of typical thin-film contact electrodes at cryogenic temperature as a control experiment. Figure 10(b) shows the hysteresis in magnetization $M\left(\mu_{0} H_{\|}\right)$ of narrow (bright orange) and wide (dark green) rectangles during up- and downsweep of an external magnetic field parallel to the strip axis. The signal was detected using a commercial superconducting quantum interference device (SQUID) magnetometer. To obtain a sizable magnetic moment, arrays of several ten thousand identical strips were EBL-patterned, as shown in the insets of Fig. 10(b).

The curves clearly show more features than the sharp and rectangular hysteresis which would be expected from an ideal single domain switching event along a magnetically easy axis. The multiple features suggest multiple domain switching, but may also stem partially from end domain con- 


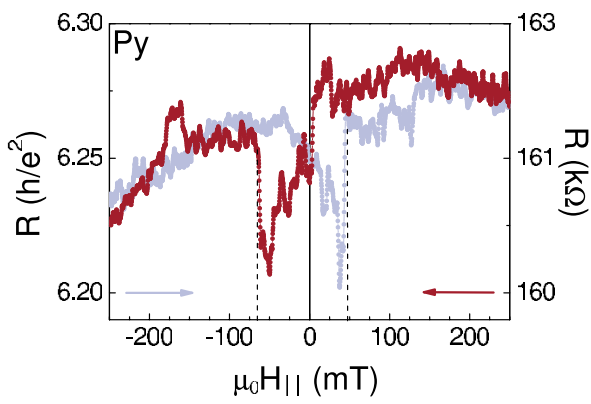

FIG. 12. (Color online) Differential two-terminal resistance $R=d V / d I$ of a MWCNT pseudo-spin valve with Py electrodes as a function of an in-plane magnetic field $B_{\|}$along the electrode long axis. The two electrodes measure $300 \mathrm{~nm} \times 16 \mu \mathrm{m}$ and $600 \mathrm{~nm} \times 16 \mu \mathrm{m}$ with a thickness of $45 \mathrm{~nm}$. The in-plane magnetic field was aligned along the length of the electrodes. The data shown were measured at $V_{g}=350 \mathrm{mV}, V_{\mathrm{sd}}=100 \mathrm{mV}$, and $T=4.2 \mathrm{~K}$. For each upsweep (in the interval [11 mT, $50 \mathrm{mT}]$ ) and downsweep (in the interval $[-11 \mathrm{mT},-69 \mathrm{mT}]$ ), a negative TMR feature of $-1 \%$ appears.

figurations, which do not impact the spin injection and detection in the tube (see, e.g., the Lorentz microscopy images of Fig. 4). Still, when comparing the SQUID data with the magnetotransport measurements, as indicated by the dashed dark green and bright orange lines in Fig. 10, the switching of magnetoresistance for both sweep directions can be matched to the sharp features in the magnetization curves. This is consistent with our findings from Lorentz microscopy that the wide electrode (dark green) has a lower coercive field than the narrow one (bright orange) by shape anisotropy.

\section{TMR in a perpendicular magnetic field}

Figure 11 displays an exemplary magnetoresistance downsweep on the same sample, as shown in Fig. 10, in a different cooling cycle, now applying a magnetic field perpendicular to the sample plane. The resulting magnetoresistance curve displays a lower noise level and a clearer TMR feature with steeper flanks of up to $3 \%$ is observed. This finding stands in contradiction to the assumption that the out-of-plane magnetization component is negligible in thin ferromagnetic films. It is consistent, however, with our observation of cross-tie domain walls in these films in Lorentz microscopy, hinting at a non-negligible out-of-plane magnetization. The switching is sharper than in parallel field, suggesting that the strips flip from one single-domain state to the other with fewer or no intermediate multi- or ripple domain states. The easy out-of-plane magnetic axis may also be the cause for the higher noise level in parallel field magnetoresistance sweeps due to a less well-defined and potentially fluctuating domain configuration.

\section{Low-temperature magnetoresistance measurements on MWCNTs}

\section{TMR in a parallel magnetic field}

An example of the two-terminal resistance of a MWCNT pseudo-spin valve with Py electrodes is shown in Fig. 12. The differential resistance is plotted as a function of magnetic field parallel to the length of the electrodes. The measurement displays negative TMR features of about $-1 \%$ for
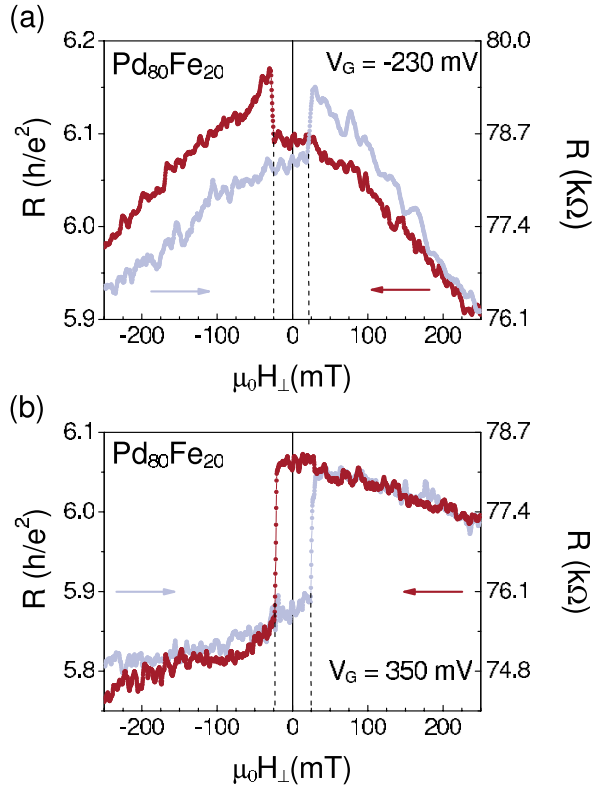

FIG. 13. (Color online) (a) Differential two-terminal resistance $R=d V / d I$ of a MWCNT pseudo-spin valve as a function of an out-of-plane magnetic field $B_{\perp}$, applying an ac bias of $\mathrm{V}_{\mathrm{sd}}=100 \mathrm{mV}$ and a gate voltage of $V_{g}=$ $-230 \mathrm{mV}$ at $4.2 \mathrm{~K}$. To access a wider range of energy levels, the pseudospin valve was patterned on a strongly coupled $\mathrm{Al}$ backgate with the about 13-nm-thick native oxide serving as dielectric. The two $\mathrm{Pd}_{80} \mathrm{Fe}_{20}$ electrodes measure $300 \mathrm{~nm} \times 16 \mu \mathrm{m}$ and $600 \mathrm{~nm} \times 16 \mu \mathrm{m}$ with a thickness of 45 $\mathrm{nm}$. A resistance jump of $1.2 \%$ occurs in downsweep (in the interval [ -23 $\mathrm{mT},-69 \mathrm{mT}]$ ) and upsweep (in the interval [20 mT, $68 \mathrm{mT}]$ ). The backswitching from antiparallel to parallel magnetization configuration is gradual rather than pronouncedly steplike. (b) Differential two-terminal resistance $R=d V / d I$ of the same sample as measured in (a) at a gate voltage of $V_{g}=350 \mathrm{mV}$. At $\pm 25 \mathrm{mT}$ for down- and upsweep respectively, a single magnetoresistance step of $3.1 \%$ occurs.

both up- and downsweep within the intervals [-11 mT, -69 $\mathrm{mT}]$ and [11 $\mathrm{mT}, 50 \mathrm{mT}]$. As observed in many of our Py or $\mathrm{Pd}_{60} \mathrm{Fe}_{40} / \mathrm{Fe}$ samples, the measurement suffers from a high noise level. Unlike the clear TMR feature of Fig. 12, in many devices random telegraph noise was observed, where the resistance switched sharply between two or more distinct values. These jumps were irreproducible in subsequent magnetosweeps and typically higher in magnitude than the TMR features, limiting the reproducibility of the magnetoresistance curves.

\section{TMR in a perpendicular magnetic field}

Two exemplary magnetoresistance sweeps of a MWCNT pseudo-spin valve as a function of an out-of-plane magnetic field are presented in Fig. 13. Also here an out-of-plane field is found to cause significant magnetoresistance effects. Figure 13(a), recorded at $V_{g}=-230 \mathrm{mV}$, displays a hysteretical behavior in the sense that at $\pm 23 \mathrm{mT}$ there is a sharp increase in resistance for up- and downsweep. The return from this positive TMR feature in antiparallel electrode magnetization alignment down to the resistance in parallel alignment is gradual indicating a gradual shift in domain walls rather than sharp switching.

In contrast, Fig. 13(b), recorded at $V_{g}=+350 \mathrm{mV}$, exhibits only a single switching event per sweep at $\pm 25 \mathrm{mT}$. Upand downsweep are still hysteretical but no longer symmetric with respect to the $B=0$ axis. The resistance increases for the 
upsweep and decreases for the downsweep between two distinct resistance levels. Although it occurs at roughly the same magnetic field value as the first switching event in (a), this behavior cannot be explained with a change in resistance due to a simple realignment of two ferromagnetic electrodes from parallel to antiparallel magnetization. The symmetric occurrence at $\pm 25 \mathrm{mT}$ hints that magnetic switching may stem from only one of the ferromagnetic electrodes, whereas in the other electrode, the magnetization is pinned probably by lattice defects, e.g., grain boundaries. So far the origin of the apparent gate dependence in Fig. 13 cannot be accounted for. It may be a specific feature of MWCNTs since it was not observed yet in SWCNTs. ${ }^{5,10}$

\section{CONCLUSIONS}

In conclusion, we have investigated various aspects of carbon nanotube-based pseudo-spin valves with diluted ferromagnetic contacts. Ferromagnetic contact electrodes of different shapes and materials were studied for their suitability as contacts for carbon-nanotube based pseudo-spin valves using TEM Lorentz microscopy. This allowed the identification of materials and shapes that are in a magnetic single domain state and whose magnetization switches at a sharp, reproducible and shape-tunable coercive field.

Permalloy structures displayed the required singledomain magnetization reversal. In comparison, PdFe alloy strips switched their magnetization via multidomain configurations. This can account for the comparatively lower reproducibility in magnetization reversal. Furthermore, cross-tie domain walls were observed as indication of a nonnegligible out-of-plane magnetization. Multiple fine ripple domains appeared in magnetic double layer structures made from $\mathrm{PdFe} / \mathrm{Fe}$.

For all electrode shapes investigated, it holds that a larger width lowers the coercive field. End domains were identified to act as seeds for magnetization reversal and thereby to decrease the coercive field. Out of the $\mathrm{Pd}_{60} \mathrm{Fe}_{40} / \mathrm{Fe}$ electrode shapes investigated here, rectangular strips exhibited the most reliable magnetization reversal, although a more pronounced end domain structure was observed compared to pointy and circular end shapes. Large-area ferromagnetic appendices to electrode strips, as in the case of attached disks or supply lines, were found to strongly impact the magnetic switching and to lead to unpredictable behavior.

Regarding the contact resistance to nanotubes, the properties of PdFe alloys were found to be significantly better than Py. Device resistances still varied strongly even along the same nanotube. SQUID measurements on large ensembles of same-sized electrode structures confirmed the coercive field scale.

Consistent with previous findings both positive and negative TMR features ${ }^{5,10}$ were observed. Using PdFe alloy electrodes of $45 \mathrm{~nm}$ thickness, we were able to observe TMR for both in-plane and out-of-plane magnetic field directions, confirming the findings from Lorentz microscopy that the out-of-plane magnetization is non-negligible in such contacts.

The giant paramagnet palladium seems to be the obvious base material for carbon nanotube-based spintronics devices. Yet, the thin film magnetic properties of different ferromagnet-Pd alloys can vary strongly, in particular, concerning the out-of-plane magnetization, and require further optimization.

\section{ACKNOWLEDGMENTS}

We would like to thank H. S. J. van der Zant, A. Morpurgo, and B. Witkamp for helping us set up our CVD system, L. Forro and C. Miko for providing the multi-walled carbon nanotubes, and M. Aprili for fruitful discussion. Thanks also go to E. Lipp for proofreading. This work was supported by the DFG within the SFB 689 and the EU FP6 CARDEQ project.

${ }^{1}$ G. A. Prinz, Science 282, 1660 (1998).

${ }^{2}$ A. Cottet, T. Kontos, S. Sahoo, H. T. Man, M. S. Choi, W. Belzig, C. Bruder, A. F. Morpurgo, and C. Schönenberger, Semicond. Sci. Technol. 21, S78 (2006).

${ }^{3}$ J. R. Hauptmann, J. Paaske, and P. E. Lindelof, Nat. Phys. 4, 373 (2008). ${ }^{4}$ M. R. Buitelaar, A. Bachtold, T. Nussbaumer, M. Iqbal, and C. Schönenberger, Phys. Rev. Lett. 88, 156801 (2002).

${ }^{5}$ H. T. Man, I. J. W. Wever, and A. F. Morpurgo, Phys. Rev. B 73, 241401(R) (2006).

${ }^{6}$ A. Javey, J. Guo, Q. Wang, M. Lundstrom, and H. Dai, Nature (London) 424, 654 (2003).

${ }^{7}$ W. G. Stirling, R. A. Cowley, and M. W. Stringfellow, J. Phys. F: Met. Phys. 2, 421 (1972).

${ }^{8}$ S. Sahoo, T. Kontos, C. Schönenberger, and C. Sürgers, Appl. Phys. Lett. 86, 112109 (2005).

${ }^{9}$ B. Zhao, I. Mönch, T. Mühl, H. Vinzelberg, and C. M. Schneider, J. Appl. Phys. 91, 7026 (2002).

${ }^{10}$ S. Sahoo, T. Kontos, J. Furer, C. Hoffmann, M. Graber, A. Cottet, and C. Schönenberger, Nat. Phys. 1, 99 (2005).

${ }^{11}$ M. Julliere, Phys. Lett. A 54, 225 (1975).

${ }^{12}$ J.-R. Kim, H. M. So, J.-J. Kim, and J. Kim, Phys. Rev. B 66, 233401 (2002).

${ }^{13}$ J. Chapman, J. Magn. Magn. Mater. 200, 729 (1999)

${ }^{14}$ C. Lim, J. Magn. Magn. Mater. 238, 301 (2002).

${ }^{15}$ T. Uhlig and J. Zweck, Phys. Rev. Lett. 93, 047203 (2004).

${ }^{16}$ A. Hubert and R. Schäfer, Magnetic Domains, 1st ed. (Springer, New York, 1998).

${ }^{17}$ R. D. Gomez, T. V. Luu, A. O. Pak, K. J. Kirk, and J. N. Chapman, J. Appl. Phys. 85, 6163 (1999).

${ }^{18}$ E. E. Shalyguina, K. H. Shin, and N. M. Abrosimova, J. Magn. Magn. Mater. 239, 252 (2002).

${ }^{19}$ J. Yu, U. Rüdiger, L. Thomas, S. S. P. Parkin, and A. D. Kent, J. Appl. Phys. 85, 5501 (1999).

${ }^{20}$ K. J. Kirk, J. N. Chapman, and C. D. W. Wilkinson, J. Appl. Phys. 85, 5237 (1999).

${ }^{21}$ M. Huber (2005) (unpublished).

${ }^{22}$ M. R. McCartney and D. J. Smith, Scanning Microsc. 11, 335 (1997).

${ }^{23}$ J. Kong, H. T. Soh, A. M. Cassell, C. F. Quate, and H. Dai, Nature (London) 395, 878 (1998). 